\title{
RATES OF CONVERGENCE FOR EMPIRICAL SPECTRAL MEASURES: A SOFT APPROACH
}

\author{
ELIZABETH S. MECKES AND MARK W. MECKES
}

\begin{abstract}
Understanding the limiting behavior of eigenvalues of random matrices is the central problem of random matrix theory. Classical limit results are known for many models, and there has been significant recent progress in obtaining more quantitative, nonasymptotic results. In this paper, we describe a systematic approach to bounding rates of convergence and proving tail inequalities for the empirical spectral measures of a wide variety of random matrix ensembles. We illustrate the approach by proving asymptotically almost sure rates of convergence of the empirical spectral measure in the following ensembles: Wigner matrices, Wishart matrices, Haar-distributed matrices from the compact classical groups, powers of Haar matrices, randomized sums and random compressions of Hermitian matrices, a random matrix model for the Hamiltonians of quantum spin glasses, and finally the complex Ginibre ensemble. Many of the results appeared previously and are being collected and described here as illustrations of the general method; however, some details (particularly in the Wigner and Wishart cases) are new.

Our approach makes use of techniques from probability in Banach spaces, in particular concentration of measure and bounds for suprema of stochastic processes, in combination with more classical tools from matrix analysis, approximation theory, and Fourier analysis. It is highly flexible, as evidenced by the broad list of examples. It is moreover based largely on "soft" methods, and involves little hard analysis.
\end{abstract}

The most fundamental problem in random matrix theory is to understand the limiting behavior of the empirical spectral distribution of large random matrices, as the size tends to infinity. The first result on this topic is the famous Wigner semi-circle law, the first version of which was proved by Wigner in 1955 [52, 53]. A random matrix is called a Wigner matrix if it is Hermitian, with independent entries on and above the diagonal. Wigner showed that, under some conditions on the distributions of the entries, the limiting empirical spectral measure of a (normalized) Wigner matrix is the semi-circular law $\rho_{s c}$.

Wigner's first version of the semi-circle law gave convergence in expectation only; i.e., he showed that the expected number of eigenvalues of a Wigner matrix in an interval converged to the value predicted by the semi-circle law, as the size of the matrix tended to infinity. His second paper improved this to convergence "weakly in probability". The analog for random unitary matrices, namely that their spectral measures converge to the uniform measure on the circle, seems intuitively obvious; surprisingly, convergence in mean and weak convergence in probability were not proved until nearly 40 years after Wigner's original work [9].

While these results are fundamental, the limitations of limit theorems such as these are well known. Just as the Berry-Esseen theorem and Hoeffding-type inequalities provide real tools for applications where the classical central limit theorem only justifies heuristics, it is essential to improve the classical limit results of random matrix theory to quantitative approximation results which have content for large but finite random matrices. See [8, 49] for extended discussions of this so-called "non-asymptotic" random matrix theory and its applications. 
In this paper, we describe a systematic approach to bounding rates of convergence and proving tail inequalities for the empirical spectral measures of a wide variety of random matrix ensembles. This approach makes use of techniques from probability in Banach spaces, in particular concentration of measure and bounds for suprema of stochastic processes, in combination with more classical tools from matrix analysis, approximation theory, and Fourier analysis. Our approach is highly flexible, and can be used for a wide variety of types of matrix ensembles, as we will demonstrate in the following sections. Moreover, it is based largely on "soft" methods, and involves little hard analysis. Our approach is restricted to settings in which there is a concentration of measure phenomenon; in this sense, it has rather different strengths than the methods used in, for example, [13, 17, 47] and many other works referred to in those papers. Those approaches achieve sharper results without requiring a measure concentration hypothesis, but they require many delicate estimates and are mainly restricted to random matrices constructed from independent random variables, whereas our methods have no independence requirements.

The following key observation, a consequence of the classical Hoffman-Wielandt inequality (see [2, Theorem VI.4.1]), underlies the approach.

Lemma 1 (see [36, Lemma 2.3]). For an $n \times n$ normal matrix $M$ over $\mathbb{C}$, let $\lambda_{1}, \ldots, \lambda_{n}$ denote the eigenvalues, and let $\mu_{M}$ denote the spectral measure of $M$; i.e.,

$$
\mu_{M}:=\frac{1}{n} \sum_{j=1}^{n} \delta_{\lambda_{j}} .
$$

Then

(a) if $f: \mathbb{C} \rightarrow \mathbb{R}$ is 1-Lipschitz, then the map

$$
M \longmapsto \int f d \mu_{M}
$$

is $\frac{1}{\sqrt{n}}$-Lipschitz, with respect to the Hilbert-Schmidt distance on the set of normal matrices; and

(b) if $\nu$ is any probability measure on $\mathbb{C}$ and $p \in[1,2]$, the map

$$
M \longmapsto W_{p}\left(\mu_{M}, \nu\right)
$$

is $\frac{1}{\sqrt{n}}$-Lipschitz.

Here $W_{p}$ denotes the $L_{p}$-Kantorovich (or Wasserstein) distance on probability measures on $\mathbb{C}$, defined by

$$
W_{p}(\mu, \nu)=\left(\inf _{\pi} \int|x-y|^{p} d \pi(x, y)\right)^{1 / p},
$$

where the infimum ranges over probability measures $\pi$ on $\mathbb{C} \times \mathbb{C}$ with marginals $\mu$ and $\nu$. The Kantorovich-Rubinstein theorem (see [50, Theorem 1.14]) gives that

$$
W_{1}(\mu, \nu)=\sup _{|f|_{L} \leq 1}\left(\int f d \mu-\int f d \nu\right),
$$

where $|f|_{L}$ denotes the Lipschitz constant of $f$; this connects part (a) of Lemma 1 with estimates on $W_{1}$.

In many random matrix ensembles of interest there is a concentration of measure phenomenon, meaning that well-behaved functions are "essentially constant", in the sense that 
they are close to their means with high probability. A prototype is the following Gaussian concentration phenomenon (see [28]).

Proposition 2. If $F: \mathbb{R}^{n} \rightarrow \mathbb{R}$ is a 1-Lipschitz function and $Z$ is a standard Gaussian random vector in $\mathbb{R}^{n}$, then

$$
\mathbb{P}[F(Z)-\mathbb{E} F(Z) \geq t] \leq e^{-t^{2} / 2}
$$

for all $t>0$.

Suppose now that $M$ is a random matrix satisfying such a concentration property. Lemma 1 means that one can obtain a bound on $W_{p}\left(\mu_{M}, \nu\right)$ which holds with high probability if one can bound $\mathbb{E} W_{p}\left(\mu_{M}, \nu\right)$. That is, a bound on the expected distance to the limiting measure immediately implies an asymptotically almost sure bound. The tail estimates coming from measure concentration are typically exponential or better, and therefore imply almost sure convergence rates via the Borel-Cantelli lemma.

We are thus left with the problem of bounding the expected distance from the empirical spectral measure $\mu_{M}$ to some deterministic reference measure $\nu$. There are two different methods used for this step, depending on the properties of the ensemble:

(1) Eigenvalue rigidity. In some ensembles, each of the (ordered) individual eigenvalues can be assigned a predicted location based on the limiting spectral measure for the ensemble, such that all (or at least many) eigenvalues concentrate strongly near these predicted locations. In this case $\nu$ is taken to be a discrete measure supported on those predicted locations, and the concentration allows one to easily estimate $\mathbb{E} W_{p}\left(\mu_{M}, \nu\right)$.

(2) Entropy methods. If instead we set $\nu=\mathbb{E} \mu_{M}$, then the Kantorovich-Rubinstein theorem implies that

$$
W_{1}\left(\mu_{M}, \nu\right)=\sup _{|f|_{L} \leq 1}\left(\int f d \mu_{M}-\mathbb{E} \int f d \mu_{M}\right),
$$

so that $W_{1}\left(\mu_{M}, \nu\right)$ is the supremum of a centered stochastic process indexed by the unit ball of the space of Lipschitz functions on $\mathbb{C}$. In ensembles with a concentration phenomenon for Lipschitz functions, part (a) of Lemma 1 translates to an increment condition on this stochastic process, which gives a route to bounding its expected supremum via classical entropy methods.

Finally, it may still be necessary to estimate the distance from the measure $\nu$ to the limiting spectral measure for the random matrix ensemble. The techniques used to do this vary by the ensemble, but this is a more classical problem of convergence of a sequence of deterministic measures to a limit, and any of the many techniques for obtaining rates of convergence may be useful.

Applications of concentration of measure to random matrices date from at least as long ago as the 1970s; a version of the argument for the concentration of $W_{1}\left(\mu_{M}, \nu\right)$ essentially appears in the 2000 paper [22] of Guionnet and Zeitouni. See [8, 29, 48, for surveys of concentration methods in random matrix theory.

The method of eigenvalue rigidity to bound Kantorovich distances is particularly suited to situations in which the empirical spectrum is a determinantal point process; this was first observed in the work of Dallaporta [6, 7]. The entropy approach to random Kantorovich distances was introduced in the context of random projections in [33, 34; it was first applied for empirical spectral measures in [35, 36]. A further abstraction was given by Ledoux [30]. 
Organization. The rest of this paper is a series of sections sketching some version of the program described above for a number of random matrix ensembles. Sections 1 and section 2 discusses Wigner and Wishart matrices, combining eigenvalue rigidity arguments of Dallaporta [6, 7] with measure concentration. Section 3 discusses random matrices drawn uniformly from classical compact matrix groups, and Section 4 discusses powers of such matrices; both those sections follow [37] and also use the eigenvalue rigidity approach. The next three sections use the entropy method: Sections 5 and 6 discusses randomized sums and random compressions of Hermitian matrices, following [36], and Section 7 discusses Hamiltonians of quantum spin glasses, following [3]. Finally, Section 8, following [38, demonstrates in case of the complex Ginibre ensemble, how eigenvalue rigidity alone allows one to carry our much of our program even without the use of a general concentration phenomenon together with Lemma 1 .

\section{Wigner matrices}

In this section we outline how our approach can be applied to the most central model of random matrix theory, that of Wigner matrices. We begin with the most classical case: the Gaussian Unitary Ensemble (GUE). Let $M_{n}$ be a random $n \times n$ Hermitian matrix, whose entries $\left\{\left[M_{n}\right]_{j k} \mid 1 \leq j \leq k \leq n\right\}$ are independent random variables, such that each $\left[M_{n}\right]_{j j}$ has a $N\left(0, n^{-1}\right)$ distribution, and each $\left[M_{n}\right]_{j k}$ for $j<k$ has independent real and imaginary parts, each with a $N\left(0,(2 n)^{-1}\right)$ distribution. Since $M_{n}$ is Hermitian, it has real eigenvalues $\lambda_{1} \leq \cdots \leq \lambda_{n}$. Wigner's theorem implies that the empirical spectral measure

$$
\mu_{n}=\frac{1}{n} \sum_{j=1}^{n} \delta_{\lambda_{j}}
$$

converges to the semicircle law $\rho_{s c}$. The following result quantifies this convergence.

Theorem 3. Let $M_{n}$ be as above, and let $\mu_{n}$ denote its spectral measure. Then

(a) $\mathbb{E} W_{2}\left(\mu_{n}, \rho_{s c}\right) \leq C \frac{\sqrt{\log (n)}}{n}$,

(b) $\mathbb{P}\left[W_{2}\left(\mu_{n}, \rho_{s c}\right) \geq C \frac{\sqrt{\log (n)}}{n}+t\right] \leq e^{-n^{2} t^{2} / 2}$ for all $t \geq 0$, and

(c) with probability 1, for sufficiently large $n, W_{2}\left(\mu_{n}, \rho_{s c}\right) \leq C^{\prime} \frac{\sqrt{\log (n)}}{n}$.

Here and in what follows, symbols such as $c, C, C^{\prime}$ denote constants which are independent of dimension.

Part (a) of Theorem 3 was proved by Dallaporta in [6] using the eigenvalue rigidity approach; the proof is outlined below.

Lemma 1 and the Gaussian concentration of measure property (Proposition 2), imply that if $F$ is a 1-Lipschitz function (with respect to the Hilbert-Schmidt distance) on the space of Hermitian matrices, then

$$
\mathbb{P}\left[F\left(M_{n}\right) \geq \mathbb{E} F\left(M_{n}\right)+t\right] \leq e^{-n t^{2} / 2}
$$

for all $t \geq 0$. This fact, together with part (b) of Lemma 1 and part (a) of Theorem 3 now imply part (b). Finally, part (c) follows from part (b) by the Borel-Cantelli lemma. So it remains only to prove part (a). 
Define $\gamma_{j} \in \mathbb{R}$ such that $\rho_{s c}\left(\left(-\infty, \gamma_{j}\right]\right)=\frac{j}{n}$; this is the predicted location of the $j^{\text {th }}$ eigenvalue $\lambda_{j}$ of $M_{n}$. The discretization $\nu_{n}$ of the semi-circle law $\rho_{s c}$ is given by

$$
\nu_{n}:=\frac{1}{n} \sum_{j=1}^{n} \delta_{\gamma_{j}} .
$$

It can be shown that that $W_{2}\left(\rho_{s c}, \nu_{n}\right) \leq \frac{C}{n}$. Furthermore, by the definition of $W_{2}$,

$$
\mathbb{E} W_{2}^{2}\left(\mu_{n}, \nu_{n}\right) \leq \frac{1}{n} \sum_{j=1}^{n} \mathbb{E}\left|\lambda_{j}-\gamma_{j}\right|^{2} .
$$

This reduces the proof of part (a) to estimating the latter expectations.

It is a classical fact that the eigenvalues of the GUE form a determinantal point process with kernel

$$
K_{n}(x, y)=\sum_{j=0}^{n} h_{j}(x) h_{j}(y) e^{-\left(x^{2}+y^{2}\right) / 2},
$$

where the $h_{j}$ are the orthonormalized Hermite polynomials [39, Section 6.2]. (The reader is referred to 24 for the definition of a determinantal point process.) The following is a then a special case of some important general properties of determinantal point processes [24, Theorem 7], [23].

Proposition 4. For each $x \in \mathbb{R}$, let $\mathcal{N}_{x}$ denote the number of eigenvalues of $M_{n}$ which are less than or equal to $x$. Then

$$
\mathcal{N}_{x} \stackrel{d}{=} \sum_{i=1}^{n} \xi_{i}
$$

where the $\xi_{i}$ are independent $\{0,1\}$-valued Bernoulli random variables.

Moreover,

$$
\mathbb{E} \mathcal{N}_{x}=\int_{-\infty}^{x} K_{n}(u, u) d u \quad \text { and } \quad \operatorname{Var} \mathcal{N}_{x}=\int_{-\infty}^{x} \int_{x}^{\infty} K_{n}(u, v)^{2} d u d v .
$$

The first part of this result can be combined with the classical Bernstein inequality to deduce that for each $t>0$,

$$
\mathbb{P}\left[\left|\mathcal{N}_{x}-\mathbb{E} \mathcal{N}_{x}\right|>t\right] \leq 2 \exp \left(-\frac{t^{2}}{2 \sigma_{x}^{2}+t}\right)
$$

where $\sigma_{x}^{2}=\operatorname{Var} \mathcal{N}_{x}$. Using estimates on $\mathbb{E} \mathcal{N}_{x}$ due to Götze and Tikhomirov [18] and on $\sigma_{x}^{2}$ due to Gustavsson [23] (both of which can be deduced from the second part of Proposition 4), this implies that for $x \in(-2+\delta, 2-\delta)$,

$$
\mathbb{P}\left[\left|\mathcal{N}_{x}-n \rho_{s c}((-\infty, x])\right|>t+C\right] \leq 2 \exp \left(-\frac{t^{2}}{2 c_{\delta} \log (n)+t}\right)
$$

for each $t \geq 0$. Combining this with the observation that

$$
\mathbb{P}\left[\lambda_{j}>\gamma_{j}+t\right]=\mathbb{P}\left[\mathcal{N}_{\gamma_{j}+t}<j\right],
$$

one can deduce, upon integrating by parts, that

$$
\mathbb{E}\left|\lambda_{j}-\gamma_{j}\right|^{2} \leq C_{\varepsilon} \frac{\log (n)}{n^{2}}
$$


for $j \in[\varepsilon n,(1-\varepsilon) n]$. This provides the necessary estimates in the bulk of the spectrum. Dallaporta established similar but weaker bounds for the soft edge of the spectrum using essentially the last part of Proposition 4, and for the hard edge using tail estimates due to Ledoux and Rider [31]. This completes the proof of Theorem 3 .

The real symmetric counterpart of the GUE is the Gaussian Orthogonal Ensemble (GOE), whose entries $\left\{\left[M_{n}\right]_{j k} \mid 1 \leq j \leq k \leq n\right\}$ are independent real random variables, such that each $\left[M_{n}\right]_{j j}$ has a $N\left(0, n^{-1}\right)$ distribution, and each $\left[M_{n}\right]_{j k}$ for $j<k$ has a $N\left(0,(\sqrt{2} n)^{-1}\right)$ distribution. The spectrum of the GOE does not form a determinantal point process, but a close distributional relationship between the eigenvalue counting functions of the GOE and GUE was found in [16, 41]. Using this, Dallaporta showed that part (a) of Theorem 3 also applies to the GOE. Part (b) then follows from the Gaussian concentration of measure property as before, and part (c)] from the Borel-Cantelli lemma.

To move beyond the Gaussian setting, Dallaporta invokes the Tao-Vu four moment theorem [46, 45] and a localization theorem due to Erdős, Yau, and Yin [14] to extend Theorem 3(a) to random matrices with somewhat more general entries. The proofs of these results involve the kind of hard analysis which it is our purpose to avoid in this paper. However, it is straightforward, under appropriate hypotheses, to extend the measure concentration argument for part (b)] of Theorem 3, and we indicate briefly how this is done.

A probability measure $\mu$ on $\mathbb{R}$ is said to satisfy a quadratic transportation cost inequality (QTCI) with constant $C>0$ if

$$
W_{2}(\mu, \nu) \leq \sqrt{C H(\nu \mid \mu)}
$$

for any probability measure $\nu$ which is absolutely continuous with respect to $\mu$, where $H(\nu \mid \mu)$ denotes relative entropy.

Proposition 5 (see [28, Chapter 6]). Suppose that $X_{1}, \ldots, X_{n}$ are independent random variables whose distributions each satisfy a QTCI with constant $C$. If $F: \mathbb{R}^{n} \rightarrow \mathbb{R}$ is a 1-Lipschitz function, then

$$
\mathbb{P}[F(X)-\mathbb{E} F(X) \geq t] \leq e^{-t^{2} / C}
$$

for all $t>0$.

A QTCI is the most general possible hypothesis which implies subgaussian tail decay, independent of $n$, for Lipschitz functions of independent random variables; see [19]. It holds in particular for any distribution satisfying a logarithmic Sobolev inequality, including Gaussian distributions, or a distribution with a density on a finite interval bounded above and below by positive constants. Using Dallaporta's arguments for part (a) and substituting Proposition 5in place of the Gaussian concentration phenomenon, we arrive at the following generalization of Theorem 3 .

Theorem 6. Let $M_{n}$ be a random Hermitian matrix whose entries satisfy each of the following:

- The random variables $\left\{\operatorname{Re} M_{j k}\right\}_{1 \leq j \leq k \leq n}$ and $\left\{\operatorname{Im} M_{j k}\right\}_{1 \leq j<k \leq n}$ are all independent.

- The first four moments of each of these random variables is the same as for the GUE (respectively, GOE).

- Each of these random variables satisfies a QTCI with constant cn ${ }^{-1 / 2}$.

Let $\mu_{n}$ denote the spectral measure of $M_{n}$. Then 
(a) $\mathbb{E} W_{2}\left(\mu_{n}, \rho_{s c}\right) \leq C \frac{\sqrt{\log (n)}}{n}$,

(b) $\mathbb{P}\left[W_{2}\left(\mu_{n}, \rho_{s c}\right) \geq C \frac{\sqrt{\log (n)}}{n}+t\right] \leq e^{-c n^{2} t^{2}}$ for all $t \geq 0$, and

(c) with probability 1 , for sufficiently large $n, W_{2}\left(\mu_{n}, \rho_{s c}\right) \leq C^{\prime} \frac{\sqrt{\log (n)}}{n}$.

As mentioned above, a QTCI is a minimal assumption to reach exactly this result by these methods. A weaker and more classical assumption would be a Poincaré inequality, which implies subexponential decay for Lipschitz functions, and is the most general hypothesis implying any decay independent of $n$; see 20] and the references therein. If the third condition in Theorem 6 is replaced by the assumption of a Poincaré inequality with constant $c n^{-1 / 2}$, then the same kind of argument leads to an almost sure convergence rate of order $\frac{\log (n)}{n}$; we omit the details.

\section{WISHART MATRICES}

In this section we apply the strategy described in the introduction to Wishart matrices (i.e., random sample covariance matrices). Let $m \geq n$, and let $X$ be an $m \times n$ random matrix with i.i.d. entries, and define the Hermitian positive-semidefinite random matrix

$$
S_{m, n}:=\frac{1}{m} X^{*} X
$$

We denote the eigenvalues of $S_{m, n}$ by $0 \leq \lambda_{1} \leq \cdots \leq \lambda_{n}$ and the empirical spectral measure by

$$
\mu_{m, n}=\frac{1}{n} \sum_{j=1}^{n} \delta_{\lambda_{j}}
$$

It was first proved in 32 that, under some moment conditions, if $\frac{n}{m} \rightarrow \rho>0$ as $n, m \rightarrow$ $\infty$, then $\mu_{m, n}$ converges to the Marchenko-Pastur law $\mu_{\rho}$ with parameter $\rho$, with compactly supported density given by

$$
f_{\rho}(x)=\frac{1}{2 \pi x} \sqrt{\left(b_{\rho}-x\right)\left(x-a_{\rho}\right)},
$$

on $\left(a_{\rho}, b_{\rho}\right)$, with $a_{\rho}=(1-\sqrt{\rho})^{2}$ and $b_{\rho}=(1+\sqrt{\rho})^{2}$. The following result quantifies this convergence for many distributions.

Theorem 7. Suppose that for each $n, 0<c \leq \frac{n}{m} \leq 1$, and that $X$ is an $m \times n$ random matrix whose entries satisfy each of the following:

- The random variables $\left\{\operatorname{Re} X_{j k}\right\}_{\substack{1 \leq j \leq m \\ 1 \leq k<n}}$ and $\left\{\operatorname{Im} X_{j k}\right\}_{\substack{1 \leq j \leq m \\ 1 \leq k \leq n}}$ are all independent.

- The first four moments of each of these random variables are the same as for a standard complex (respectively, real) normal random variable.

- Each of these random variables satisfies a QTCI with constant $C$.

Let $\rho=\frac{n}{m}$ and let $\mu_{m, n}$ denote the spectral measure of $S_{m, n}=\frac{1}{m} X^{*} X$. Then

(a) $\mathbb{E} W_{2}\left(\mu_{m, n}, \mu_{\rho}\right) \leq C \frac{\sqrt{\log (n)}}{n}$,

(b) $\mathbb{P}\left[W_{2}\left(\mu_{m, n}, \mu_{\rho}\right) \geq C \frac{\sqrt{\log (n)}}{n}+t\right] \leq e^{-c m \min \left\{n t^{2}, \sqrt{n} t\right\}}$ for all $t \geq c \frac{\sqrt{\log (n)}}{n}$, and 
(c) with probability 1, for sufficiently large $n, W_{2}\left(\mu_{m, n}, \mu_{\rho}\right) \leq C^{\prime} \frac{\sqrt{\log (n)}}{n}$.

Strictly speaking, part (c) does not, as stated, imply almost sure convergence of $\mu_{m, n}$, since $\rho$ and hence $\mu_{\rho}$ itself depends on $n$. However, if $\rho=\rho(n)$ has a limiting value $\rho^{*}$ as $n \rightarrow \infty$ (as in the original Marchenko-Pastur result), then the measures $\mu_{\rho}$ converge to $\mu_{\rho^{*}}$. This convergence can easily be quantified, but we will not pursue the details here.

Proof. Part (a) was proved by Dallaporta in [7], by the same methods as in Theorem G(a) discussed in the last section. First, when the entries of $X$ are complex normal random variables (in which $S_{m, n}$ is the unitary Laguerre ensemble), the eigenvalues of $S_{m, n}$ form a determinantal point process. This implies an analogue of Proposition 4 , from which eigenvalue rigidity results can be deduced, leading to the estimate in part (a) in this case. The result is extended to real Gaussian random matrices using interlacing results, and to more general distributions using versions of the four moment theorem for Wishart random matrices. The reader is referred to [7] for the details.

The proof of part (b) is more complicated than in the previous section, because the random matrix $S_{m, n}$ depends quadratically on the independent entries of $X$. However, we can still apply the machinery of measure concentration by using the fact that $S_{m, n}$ possesses local Lipschitz behavior, combined with a truncation argument. Indeed, if $X, Y$ are $m \times n$ matrices over $\mathbb{C}$,

$$
\begin{aligned}
\left\|\frac{1}{m} X^{*} X-\frac{1}{m} Y^{*} Y\right\|_{H S} & \left.\leq \frac{1}{m}\left\|X^{*}(X-Y)\right\|_{H S}+\frac{1}{m} \|\left(X^{*}-Y^{*}\right) Y\right) \|_{H S} \\
& \leq \frac{1}{m}\left(\|X\|_{o p}+\|Y\|_{o p}\right)\|X-Y\|_{H S},
\end{aligned}
$$

where we have used the facts that both the Hilbert-Schmidt norm $\|\cdot\|_{H S}$ and the operator norm $\|\cdot\|_{o p}$ are invariant under conjugation and transposition, and that $\|A B\|_{H S} \leq$ $\|A\|_{o p}\|B\|_{H S}$.

Thus, for a given $K>0$, the function

$$
X \mapsto \frac{1}{m} X^{*} X
$$

is $\frac{2 K}{\sqrt{m}}$-Lipschitz on $\left\{X \in \mathrm{M}_{m, n}(\mathbb{C}) \mid\|X\|_{o p} \leq K \sqrt{m}\right\}$, and so by Lemma [u[ (b), the function

$$
F: X \mapsto W_{2}\left(\mu_{m, n}, \mu_{\rho}\right)
$$

is $\frac{2 K}{\sqrt{m n}}$-Lipschitz on this set. We can therefore extend $F$ to a $\frac{2 K}{\sqrt{m n}}$-Lipschitz function $\widetilde{F}: \mathrm{M}_{m, n}(\mathbb{C}) \rightarrow \mathbb{R}($ cf. [15, Theorem 3.1.2]); we may moreover assume that $\widetilde{F}(X) \geq 0$ and

$$
\sup _{X \in \mathrm{M}_{m, n}(\mathbb{C})} \widetilde{F}(X)=\sup _{\|X\|_{o p} \leq K \sqrt{m}} W_{2}\left(\mu_{m, n}, \mu_{\rho}\right) .
$$

Proposition 5 now allows us to control $\widetilde{F}(X)$ and $\|X\|_{o p}$, which are both Lipschitz functions of $X$.

First, an elementary discretization argument using Proposition 5 (cf. [49, Theorem 5.39], or alternatively Lemma 15 below) shows that

$$
\mathbb{P}\left[\|X\|_{o p}>K \sqrt{m}\right] \leq 2 e^{-c m}
$$

for some $K, c>0$. We will use this $K$ in the following. 
Next, Proposition 5 implies that

$$
\mathbb{P}[\widetilde{F}(X)>t] \leq C e^{-c m n t^{2}}
$$

as long as $t \geq 2 \mathbb{E} \widetilde{F}(X)$. Now

$$
\begin{aligned}
\mathbb{E} \widetilde{F}(X) & =\mathbb{E} W_{2}\left(\mu_{m, n}, \mu_{\rho}\right)+\mathbb{E}\left[\left(\widetilde{F}(X)-W_{2}\left(\mu_{m, n}, \mu_{\rho}\right)\right) \mathbb{1}_{\left.\|X\|_{o p}>K \sqrt{m}\right]}\right. \\
& \leq C \frac{\sqrt{\log (n)}}{n}+\left(\sup _{\|X\|_{o p} \leq K \sqrt{m}} W_{2}\left(\mu_{m, n}, \mu_{\rho}\right)\right) \mathbb{P}\left[\|X\|_{o p}>K \sqrt{m}\right]
\end{aligned}
$$

by part (a) and (3). Since $\mu_{\rho}$ is supported on $\left[a_{\rho}, b_{\rho}\right]$, and $\mu_{m, n}$ is supported on $\left[0,\left\|\frac{1}{m} X X^{*}\right\|_{o p}\right]=$ $\left[0, \frac{1}{m}\|X\|_{o p}^{2}\right]$,

$$
\sup _{\|X\|_{o p} \leq K \sqrt{m}} W_{2}\left(\mu_{m, n}, \mu_{\rho}\right) \leq \max \left\{b_{\rho}, K^{2}\right\} \leq C,
$$

and so by (44) and (6),

$$
\mathbb{E} \tilde{F}(X) \leq C \frac{\sqrt{\log (n)}}{n}+C e^{-c m} \leq C^{\prime} \frac{\sqrt{\log (n)}}{n} .
$$

Finally, we have

$$
\begin{aligned}
\mathbb{P}\left[W_{2}\left(\mu_{m, n}, \mu_{\rho}\right)>t\right] & \leq \mathbb{P}\left[W_{2}\left(\mu_{m, n}, \mu_{\rho}\right)>t,\|X\|_{o p} \leq K \sqrt{m}\right]+\mathbb{P}\left[\|X\|_{o p}>K \sqrt{m}\right] \\
& \leq \mathbb{P}[\widetilde{F}(X)>t]+\mathbb{P}\left[\|X\|_{o p}>K \sqrt{m}\right] \\
& \leq C^{\prime} e^{-c m n t^{2}}
\end{aligned}
$$

for $c_{1} \frac{\sqrt{\log (n)}}{n} \leq t \leq \frac{c_{2}}{\sqrt{n}}$ by (4) and (5). We omit the details of the similar argument to obtain a subexponential bound for $t>\frac{c_{2}}{\sqrt{n}}$. This concludes the proof of part (b).

Part (c) follows as before using the Borel-Cantelli lemma.

An alternative approach to quantifying the limiting behavior of the spectrum of Wishart matrices is to consider the singular values $0 \leq \sigma_{1} \leq \cdots \leq \sigma_{n}$ of $\frac{1}{\sqrt{m}} X$; that is, $\sigma_{j}=\sqrt{\lambda_{j}}$. Lemma 1 can be applied directly in that context, by using the fact that the eigenvalues of the Hermitian matrix $\left[\begin{array}{cc}0 & X \\ X^{*} & 0\end{array}\right]$ are $\left\{ \pm \sigma_{j}\right\}$. However, if one is ultimately interested in the eigenvalues $\left\{\lambda_{j}\right\}$, then translating the resulting concentration estimates to eigenvalues ends up requiring the same kind of analysis carried out above.

\section{UNIFORM RANDOM MATRICES FROM THE COMPACT CLASSICAL GROUPS}

Each of the compact classical matrix groups $\mathbb{O}(n), \mathbb{S O}(n), \mathbb{U}(n), \mathbb{S U}(n), \mathbb{S} \mathbb{p}(2 n)$ possesses a uniform (Haar) probability measure which is invariant under translation by a fixed group element. Each of these uniform measures possesses a concentration of measure property making it amenable to the program laid out in the introduction; moreover, the eigenvalues of a random matrix from any of these groups is a determinantal point process, meaning that the eigenvalue rigidity approach used in Section 1 applies here as well. The limiting empirical spectral measure for all of these groups is the uniform probability measure on the 
circle, as first shown in [9]. This convergence is quantified in the following result, proved in 37.

Theorem 8. Let $M_{n}$ be uniformly distributed in any of $\mathbb{O}(n), \mathbb{S O}(n), \mathbb{U}(n), \mathbb{S U}(n)$, $\mathbb{S}_{\mathbb{P}}(2 n)$, and let $\mu_{n}$ denote its spectral measure. Let $\mu$ denote the uniform probability measure on the unit circle $\mathbb{S}^{1} \subseteq \mathbb{C}$. Then

(a) $\mathbb{E} W_{2}\left(\mu_{n}, \mu\right) \leq C \frac{\sqrt{\log (n)}}{n}$,

(b) $\mathbb{P}\left[W_{2}\left(\mu_{n}, \mu\right) \geq C \frac{\sqrt{\log (n)}}{n}+t\right] \leq e^{-c n^{2} t^{2}}$, and

(c) with probability 1, for sufficiently large $n, W_{2}\left(\mu_{n}, \mu\right) \leq C \frac{\sqrt{\log (n)}}{n}$.

We briefly sketch the proof below; for full details, see [37.

Part (a) is proved using the eigenvalue rigidity approach described in Section 1 for the GUE. We first order the eigenvalues of $M_{n}$ as $\left\{e^{i \theta_{j}}\right\}_{1 \leq j \leq n}$ with $0 \leq \theta_{1} \leq \cdots \leq \theta_{n}<2 \pi$, and define the discretization $\nu_{n}$ of $\mu$ by

$$
\nu_{n}:=\frac{1}{n} \sum_{j=1}^{n} \delta_{e^{2 \pi i j / n}}
$$

It is easy to show that $W_{2}\left(\mu, \nu_{n}\right) \leq \frac{C}{n}$, and by the definition of $W_{2}$,

$$
\mathbb{E} W_{2}^{2}\left(\mu_{n}, \nu_{n}\right) \leq \frac{1}{n} \sum_{j=1}^{n} \mathbb{E}\left|e^{i \theta_{j}}-e^{2 \pi i j / n}\right|^{2} \leq \frac{1}{n} \sum_{j=1}^{n} \mathbb{E}\left|\theta_{j}-\frac{2 \pi j}{n}\right|^{2},
$$

so that part (a) can be proved by estimating the latter expectations.

For these estimates, as for the GUE, one can make use of the determinantal structure of the eigenvalue processes of uniformly distributed random matrices. For the case of the unitary group $\mathbb{U}(n)$, the eigenvalue angles $\left\{\theta_{j}\right\}$ form a determinantal point process on $[0,2 \pi)$ with kernel

$$
K_{n}:=\frac{\sin \left(\frac{n(x-y)}{2}\right)}{\sin \left(\frac{(x-y)}{2}\right)}
$$

this was first proved by Dyson [11. The determinantal structure provides an analogue of Proposition 4

Proposition 9. For each $0 \leq x<2 \pi$, let $\mathcal{N}_{x}$ denote the number of eigenvalues $e^{i \theta_{j}}$ of $M_{n} \in \mathbb{U}(n)$ such that $\theta_{j} \leq x$. Then

$$
\mathcal{N}_{x} \stackrel{d}{=} \sum_{i=1}^{n} \xi_{i}
$$

where the $\xi_{i}$ are independent $\{0,1\}$-valued Bernoulli random variables.

Moreover,

$$
\mathbb{E} \mathcal{N}_{x}=\int_{0}^{x} K_{n}(u, u) d u \quad \text { and } \quad \operatorname{Var}_{\mathcal{N}_{x}}=\int_{0}^{x} \int_{x}^{2 \pi} \infty K_{n}(u, v)^{2} d u d v .
$$


Appropriately modified versions of Proposition 9 hold for the other groups as well, due to determinantal structures in those contexts identified by Katz and Sarnak [26].

Using (9), one can estimate $\mathbb{E} \mathcal{N}_{x}$ and $\operatorname{Var} \mathcal{N}_{x}$, and then use (8) and Bernstein's inequality to deduce that

$$
\mathbb{P}\left[\left|\mathcal{N}_{x}-\frac{n x}{2 \pi}\right|>t+C\right] \leq 2 \exp \left(-\frac{t^{2}}{2 c \log (n)+t}\right)
$$

for all $t>0$. Combining this with the observation that

$$
\mathbb{P}\left[\theta_{j}>\frac{2 \pi j}{n}+t\right]=\mathbb{P}\left[\mathcal{N}_{\frac{2 \pi j}{n}+t}<j\right],
$$

one can deduce, upon integrating by parts, that

$$
\mathbb{E}\left|\theta_{j}-\frac{2 \pi j}{n}\right|^{2} \leq C \frac{\log (n)}{n^{2}}
$$

for each $j$, which completes the proof of part (a). Observe that this is made slightly simpler than the proof of Theorem [a (a) for the GUE by the fact that all of the eigenvalues of a unitary matrix behave like "bulk" eigenvalues.

Part (b) of Theorem 8 follows from part (a) and the following concentration of measure property of the uniform measure on the compact classical groups. (There is an additional subtlety in dealing with the two components of $\mathbb{O}(n)$, which can be handled by conditioning on $\operatorname{det} M_{n}$.)

Proposition 10. Let $G_{n}$ be one of $\mathbb{S O}(n), \mathbb{U}(n), \mathbb{S U}(n)$, or $\mathbb{S p}(2 n)$, and let $F: G_{n} \rightarrow \mathbb{R}$ be 1-Lipschitz, with respect to either the Hilbert-Schmidt distance or the geodesic distance on $G_{n}$. Let $M_{n}$ be a uniformly distributed random matrix in $G_{n}$. Then

$$
\mathbb{P}\left[\left|F\left(M_{n}\right)-\mathbb{E} F\left(M_{n}\right)\right|>t\right] \leq e^{-c n t^{2}}
$$

for every $t>0$.

For $\mathbb{S O}(n), \mathbb{S U}(n)$, and $\mathbb{S p}(2 n)$, this property goes back to the work of Gromov and Milman [21; for the precise version stated here see [1, Section 4.4]. For $\mathbb{U}(n)$ (which was not covered by the results of [21] because its Ricci tensor is degenerate), the concentration in Proposition 10 was proved in [37.

Finally, part (c) follows from part (b) via the Borel-Cantelli lemma, thus completing the proof of Theorem 8 ,

\section{PoWERS OF UNIFORM RANDOM MATRICES}

The approach used with random matrices from the compact classical groups in the previous section can be readily generalized to powers of such matrices, as follows.

Theorem 11. Let $M_{n}$ be uniformly distributed in any of $\mathbb{O}(n), \mathbb{S O}(n), \mathbb{U}(n), \mathbb{S U}(n)$, $\mathbb{S p}(2 n)$. Let $m \geq 1$, and let $\mu_{m, n}$ denote the spectral measure of $M_{n}^{m}$. Let $\mu$ denote the uniform probability measure on the unit circle $\mathbb{S}^{1} \subseteq \mathbb{C}$. There are universal constants $C, c$ such that

(a) $\mathbb{E} W_{2}\left(\mu_{m, n}, \mu\right) \leq C \frac{\sqrt{m\left(\log \left(\frac{n}{m}\right)+1\right)}}{n}$, 
(b) $\mathbb{P}\left[W_{2}\left(\mu_{m, n}, \mu\right) \geq C \frac{\sqrt{m\left(\log \left(\frac{n}{m}\right)+1\right)}}{n}+t\right] \leq e^{-c n^{2} t^{2}}$, and

(c) with probability 1 , for sufficiently large $n, W_{2}\left(\mu_{m, n}, \mu\right) \leq C \frac{\sqrt{m\left(\log \left(\frac{n}{m}\right)+1\right)}}{n}$.

In fact, the same proof works for $m>1$ as in the previous section, because of the following result of Rains [42]. The result is stated in the unitary case for simplicity, but analogous results hold in the other compact classical matrix groups.

Proposition 12. Let $m \leq n$ be fixed. If $M_{n}$ is uniformly distributed in $\mathbb{U}(n)$, the eigenvalues of $M_{n}^{m}$ are distributed as those of $m$ independent uniform unitary matrices of sizes $\left\lfloor\frac{n}{m}\right\rfloor:=\max \left\{k \in \mathcal{N} \mid k \leq \frac{n}{m}\right\}$ and $\left\lceil\frac{n}{m}\right\rceil:=\min \left\{k \in \mathcal{N} \mid k \geq \frac{n}{m}\right\}$, such that the sum of the sizes of the matrices is $n$.

As a consequence, if $\mathcal{N}_{x}$ is the number of eigenvalues of $M_{n}^{m}$ lying in the arc from 1 to $e^{i x}$, then

$$
\mathcal{N}_{x} \stackrel{d}{=} \sum_{j=0}^{m-1} \mathcal{N}_{x}^{j}
$$

where the $\mathcal{N}_{\theta}^{j}$ are the counting functions of $m$ independent random matrices, each uniformly distributed in $\mathbb{U}\left(\left\lfloor\frac{n}{m}\right\rfloor\right)$ or $\mathbb{U}\left(\left\lceil\frac{n}{m}\right\rceil\right)$. In particular, by Proposition $9 \mathcal{N}_{x}$ is equal in distribution to a sum of independent Bernoulli random variables, and its mean and variance can be estimated using the available estimates for the individual summands established in the previous section. One can thus again apply Bernstein's inequality to obtain eigenvalue rigidity, leading to a bound on $\mathbb{E} W_{2}\left(\mu_{m, n}, \mu\right)$.

Crucially, the concentration phenomenon on the compact classical groups tensorizes in a dimension-free way: the product of uniform measure on the $m$ smaller unitary groups above has the same concentration property as any one of those groups. This is a consequence of the fact that the uniform measures on the compact classical groups satisfy logarithmic Sobolev inequalities; see [1, Section 4.4] and the Appendix of [37. This allows for the full program laid out in the introduction to be carried out in this case, yielding Theorem 11 above.

\section{RANDOMIZED SUMS}

In this section we show how our approach can be applied to randomized sums of Hermitian matrices. In this and the following two sections, we no longer have a determinantal structure allowing us to use eigenvalue rigidity. Instead we will use entropy methods to bound the expected distance between the empirical spectral measure and its mean.

Let $A_{n}$ and $B_{n}$ be fixed $n \times n$ Hermitian matrices, and let $U_{n} \in \mathbb{U}(n)$ be uniformly distributed. Define

$$
M_{n}:=U_{n} A_{n} U_{n}^{*}+B_{n}
$$

the random matrix $M_{n}$ is the so-called randomized sum of $A_{n}$ and $B_{n}$. This random matrix model has been studied at some length in free probability theory; the limiting spectral measure was studied first by Voiculescu [51] and Speicher [43], who showed that if $\left\{A_{n}\right\}$ and $\left\{B_{n}\right\}$ have limiting spectral distributions $\mu_{A}$ and $\mu_{B}$ respectively, then the limiting spectral distribution of $M_{n}$ is given by the free convolution $\mu_{A} \boxplus \mu_{B}$. 
The following sharpening of this convergence is a special case of Theorem 3.8 and Corollary 3.9 of [36]; we present below a slightly simplified version of the argument from that paper.

Theorem 13. In the setting above, let $\mu_{n}$ denote the empirical spectral measure of $M_{n}$, and let $\nu_{n}=\mathbb{E} \mu_{n}$. Then

(a) $\mathbb{E} W_{1}\left(\mu_{n}, \nu_{n}\right) \leq \frac{C\left\|A_{n}\right\|_{o p}^{2 / 3}\left(\left\|A_{n}\right\|_{o p}+\left\|B_{n}\right\|_{o p}\right)^{1 / 3}}{n^{2 / 3}}$,

(b) $\mathbb{P}\left[W_{1}\left(\mu_{n}, \nu_{n}\right) \geq \frac{C\left\|A_{n}\right\|_{o p}^{2 / 3}\left(\left\|A_{n}\right\|_{o p}+\left\|B_{n}\right\|_{o p}\right)^{1 / 3}}{n^{2 / 3}}+t\right] \leq e^{-c n^{2} t^{2} /\left\|A_{n}\right\|_{o p}^{2}}$, and

(c) with probability 1 , for sufficiently large $n$,

$$
W_{1}\left(\mu_{n}, \nu_{n}\right) \leq C^{\prime}\left\|A_{n}\right\|_{o p}^{2 / 3}\left(\left\|A_{n}\right\|_{o p}+\left\|B_{n}\right\|_{o p}\right)^{1 / 3} n^{-2 / 3} .
$$

In the most typical situations of interest, $\left\|A_{n}\right\|_{o p}$ and $\left\|B_{n}\right\|_{o p}$ are bounded independently of $n$. If $\left\{A_{n}\right\}$ and $\left\{B_{n}\right\}$ have limiting spectral distributions $\mu_{A}$ and $\mu_{B}$ respectively, then the rate of convergence of the (deterministic) measures $\nu_{n}$ to $\mu_{A} \boxplus \mu_{B}$ will depend strongly on the sequences $\left\{A_{n}\right\}$ and $\left\{B_{n}\right\}$; we will not address that question here.

The Lipschitz property which is a crucial ingredient of our approach to prove Theorem 13 is provided by the following lemma.

Lemma 14. For each 1-Lipschitz function $f: \mathbb{R} \rightarrow \mathbb{R}$, the maps

$$
U_{n} \mapsto \int f d \mu_{n} \quad \text { and } \quad U_{n} \mapsto W_{1}\left(\mu_{n}, \nu_{n}\right)
$$

are $\frac{2\left\|A_{n}\right\|_{o p}}{\sqrt{n}}$-Lipschitz on $\mathbb{U}(n)$.

Proof. Let $A$ and $B$ be $n \times n$ Hermitian matrices, and let $U, V \in \mathbb{U}(n)$. Then it is straightforward to show that

$$
\left\|\left(U A U^{*}+B\right)-\left(V A V^{*}+B\right)\right\|_{H S} \leq 2\|A\|_{o p}\|U-V\|_{H S}
$$

(see [36, Lemma 3.2]). The lemma now follows by Lemma 1.

Part (b) of Theorem 13 now follows from part (a) using Lemma 14 and the concentration of measure phenomenon for $\mathbb{U}(n)$ (Proposition 10), and part (c) follows as usual by the Borel-Cantelli lemma. It remains to prove part (a) as mentioned above, this is done using entropy techniques for bounding the supremum of a stochastic process.

The following lemma summarizes what is needed here. This fact is well-known to experts, but we were not able to find an explicit statement in the literature.

Lemma 15. Suppose that $(V,\|\cdot\|)$ be a finite-dimensional normed space with unit ball $\mathcal{B}(V)$, and that $\left\{X_{v} \mid v \in V\right\}$ is a family of centered random variables such that

$$
\mathbb{P}\left[\left|X_{u}-X_{v}\right| \geq t\right] \leq 2 e^{-t^{2} / K^{2}\|u-v\|^{2}}
$$

for every $t \geq 0$. Then

$$
\mathbb{E} \sup _{v \in \mathcal{B}(V)} X_{v} \leq C K \sqrt{\operatorname{dim} V}
$$


Proof. This can be proved via an elementary $\varepsilon$-net argument, but a quicker proof can be given using Dudley's entropy bound (see [44, p. 22] for a statement, and [44, p. 70] and [10] for discussions of the history of this result and its name).

By rescaling it suffices to assume that $K=1$. Let $N(\varepsilon)$ denote the number of $\varepsilon$-balls in $V$ needed to cover the unit ball $\mathcal{B}(V)$. A standard volumetric argument (see e.g. [49, Lemma 5.2]) shows that $N(\varepsilon) \leq(3 / \varepsilon)^{\operatorname{dim} V}$ for each $0<\varepsilon<1$; of course $N(\varepsilon)=1$ for $\varepsilon \geq 1$. Then Dudley's bound yields

$$
\mathbb{E} \sup _{v \in \mathcal{B}(V)} X_{v} \leq C \int_{0}^{\infty} \sqrt{\log (N(\varepsilon))} d \varepsilon \leq C \sqrt{\operatorname{dim} V} \int_{0}^{1} \sqrt{\log (3 / \varepsilon)} d \varepsilon \leq C^{\prime} \sqrt{\operatorname{dim} V} .
$$

To apply this lemma in our setting, denote by

$$
\operatorname{Lip}_{0}:=\left\{f:\left.\mathbb{R} \rightarrow \mathbb{R}|| f\right|_{L}<\infty \text { and } f(0)=0\right\},
$$

so that $\operatorname{Lip}_{0}$ is a Banach space with norm $|\cdot|_{L}$. For each $f \in \operatorname{Lip}_{0}$, define the random variable

$$
X_{f}:=\int f d \mu_{n}-\mathbb{E} \int f d \mu_{n} .
$$

By the Kantorovich-Rubinstein theorem,

$$
W_{1}\left(\mu_{n}, \nu_{n}\right)=\sup \left\{X_{f}: f \in \mathcal{B}\left(\operatorname{Lip}_{0}\right)\right\} .
$$

Lemma 14 and Proposition 10 imply that

$$
\mathbb{P}\left[\left|X_{f}-X_{g}\right| \geq t\right]=\mathbb{P}\left[\left|X_{f-g}\right| \geq t\right] \leq 2 \exp \left[-\frac{c n^{2} t^{2}}{\left\|A_{n}\right\|_{o p}^{2}|f-g|_{L}^{2}}\right] .
$$

We would like to appeal to Lemma 15, but unfortunately, Lip $_{0}$ is infinite-dimensional. We can get around this problem with an additional approximation argument.

Observing that $\mu_{n}$ is supported on $\left[-\left\|M_{n}\right\|_{o p},\left\|M_{n}\right\|_{o p}\right]$ and $\left\|M_{n}\right\|_{o p} \leq\left\|A_{n}\right\|_{o p}+\left\|B_{n}\right\|_{o p}$, we begin by replacing $\operatorname{Lip}_{0}$ with

$$
\operatorname{Lip}_{0}([-R, R]):=\left\{f:\left.[-R, R] \rightarrow \mathbb{R}|| f\right|_{L}<\infty \text { and } f(0)=0\right\}
$$

with $R=\left\|A_{n}\right\|_{o p}+\left\|B_{n}\right\|_{o p}$, for (11), (12), and (13) above. Now for an integer $m \geq 1$, let $\operatorname{Lip}_{0}^{m}([-R, R])$ be the $2 m$-dimensional space of piecewise affine functions $f \in \operatorname{Lip}_{0}([-R, R])$ such that $f$ is affine on each interval $\left[-R+\frac{(k-1) R}{m},-R+\frac{k R}{m}\right]$ for $k=1, \ldots, 2 m$. Given $f \in \operatorname{Lip}_{0}([-R, R])$, there is a unique function $g \in \operatorname{Lip}_{0}^{m}([-R, R])$ such that $g\left(\frac{j R}{m}\right)=f\left(\frac{j R}{m}\right)$ for each integer $j \in[-m, m]$; and this $g$ satisfies

$$
|g|_{L} \leq|f|_{L} \quad \text { and } \quad\|f-g\|_{\infty} \leq \frac{|f|_{L} R}{2 m}
$$

Thus by (12),

$$
W_{1}\left(\mu_{n}, \nu_{n}\right) \leq \frac{R}{2 m}+\sup \left\{X_{g} \mid g \in \mathcal{B}\left(\operatorname{Lip}_{0}^{m}([-R, R])\right)\right\} .
$$

Now by (13) and Lemma 15,

$$
\mathbb{E} W_{1}\left(\mu_{n}, \nu_{n}\right) \leq \frac{R}{2 m}+C \frac{\left\|A_{n}\right\|_{o p} \sqrt{m}}{n} .
$$

Part (a) now follows by optimizing over $m$. This completes the proof of Theorem 13 . 
An additional conditioning argument allows one to consider the case that $A_{n}$ and $B_{n}$ are themselves random matrices in Theorem 13, assuming a concentration of measure property for their distributions. We refer to [36] for details.

It seems that the entropy method does not usually result in sharp rates; for example, in [36], we used the entropy approach for Wigner and Haar-distributed matrices, and the results were not as strong as those in Sections 1 and 3 . On the other hand, the entropy method is more widely applicable than the determinantal point process methods which yielded the results of Sections 1 and 3 . In addition to the randomized sums treated in this section, we show in Sections 6 and 7 how the entropy method can be used for random compressions and for the Hamiltonians of quantum spin glasses. The paper 36] also used the entropy approach to prove convergence rates for the empirical spectral measures of the circular orthogonal ensemble and the circular symplectic ensemble, which we have omitted from this paper.

\section{RANDOM COMPRESSIONS}

Let $A_{n}$ be a fixed $n \times n$ Hermitian (respectively, real symmetric) matrix, and let $U_{n}$ be uniformly distributed in $\mathbb{U}(n)$ (respectively, $\mathbb{O}(n)$ ). Let $P_{k}$ denote the projection of $\mathbb{C}^{n}$ (respectively $\mathbb{R}^{n}$ ) onto the span of the first $k$ standard basis vectors. Finally, define a random matrix $M_{n}$ by

$$
M:=P_{k} U_{n} A_{n} U_{n}^{*} P_{k}^{*} .
$$

Then $M_{n}$ is a compression of $A_{n}$ to a random $k$-dimensional subspace. In the case that $\left\{A_{n}\right\}_{n \in \mathbb{N}}$ has a limiting spectral distribution and $\frac{k}{n} \rightarrow \alpha$, the limiting spectral distribution of $M_{n}$ can be determined using techniques of free probability (see 43]); the limit is given by a free-convolution power related to the limiting spectral distribution of $A_{n}$ and the value $\alpha$.

For this random matrix model, the program laid out in the introduction produces the following (cf. Theorem 3.5 and Corollary 3.6 in [36]).

Theorem 16. In the setting above, let $\mu_{n}$ denote the empirical spectral distribution of $M_{n}$, and let $\nu_{n}=\mathbb{E} \mu_{n}$. Then

(a) $\mathbb{E} W_{1}\left(\mu_{n}, \nu_{n}\right) \leq \frac{C\left\|A_{n}\right\|_{o p}}{\left((k n)^{1 / 3}\right.}$,

(b) $\mathbb{P}\left[W_{1}\left(\mu_{n}, \nu_{n}\right) \geq \frac{C\left\|A_{n}\right\|_{o p}}{(k n)^{1 / 3}}+t\right] \leq e^{-c k n t^{2} /\left\|A_{n}\right\|_{o p}^{2}}$, and

(c) with probability 1 , for sufficiently large $n, W_{1}\left(\mu_{n}, \nu_{n}\right) \leq C^{\prime}\left\|A_{n}\right\|_{o p}(k n)^{-1 / 3}$.

The proof is essentially identical to the one in the previous section; the $k$-dependence in the bounds is a consequence of the fact that $k$, not $n$, is the size of the matrix when Lemma 1 is applied. As with Theorem 13, an additional conditioning argument allows one to consider the case that $A_{n}$ is random, with distribution satisfying a concentration of measure property.

\section{Hamiltonians of QUANTUM SPIN GLASSES}

In this section we consider the following random matrix model for the Hamiltonian of a quantum spin glass: let $\left\{Z_{a, b, j}\right\}_{\substack{1 \leq a, b \leq 3 \\ 1 \leq j \leq n}}$ be independent standard Gaussian random variables, 
and define the $2^{n} \times 2^{n}$ random Hermitian matrix $H_{n}$ by

$$
H_{n}:=\frac{1}{\sqrt{9 n}} \sum_{j=1}^{n} \sum_{a, b=1}^{3} Z_{a, b, j} \sigma_{j}^{(a)} \sigma_{j+1}^{(b)},
$$

where for $1 \leq a \leq 3$,

$$
\sigma_{j}^{(a)}:=I_{n}^{\otimes(j-1)} \otimes \sigma^{(a)} \otimes I_{2}^{\otimes(n-j)},
$$

with $I_{2}$ denoting the $2 \times 2$ identity matrix, $\sigma^{(a)}$ denoting the $2 \times 2$ matrices

$$
\sigma^{(1)}:=\left[\begin{array}{ll}
0 & 1 \\
1 & 0
\end{array}\right] \quad \sigma^{(2)}:=\left[\begin{array}{cc}
0 & -i \\
i & 0
\end{array}\right] \quad \sigma^{(3)}:=\left[\begin{array}{cc}
1 & 0 \\
0 & -1
\end{array}\right]
$$

and the labeling cyclic so that $\sigma_{n+1}^{(b)}:=\sigma_{1}^{(b)}$. The random matrix $H_{n}$ acts on the space $\left(\mathbb{C}^{2}\right)^{\otimes n}$ of $n$ distinguishable qubits; the specific structure of $H_{n}$ above corresponds to nearest neighbor interaction on a circle of qubits.

If $\mu_{n}$ denotes the empirical spectral measure of $H_{n}$, then the ensemble average $\nu_{n}=\mathbb{E} \mu_{n}$ is known in this context as the density of states measure $\mu_{n}^{D O S}$. Recently, Keating, Linden and Wells [27] showed that $\mu_{n}^{D O S}$ converges weakly to Gaussian, as $n \rightarrow \infty$; i.e., they showed that the empirical spectral measure of $H_{n}$ converges to Gaussian in expectation. The paper [27] gives a similar treatment for more general collections of (still independent) coupling coefficients, and more general coupling geometries than that of nearest-neighbor interactions. In more recent work, Erdős and Schröder [12 have considered still more general coupling geometries, and found a sharp transition in the limiting behavior of the density of states measure depending on the size of the maximum degree of the underlying graph, relative to its number of edges.

The following result, essentially proved in [3], quantifies this convergence.

Theorem 17. Let $\mu_{n}$ be the spectral measure of $H_{n}$ and let $\gamma$ denote the standard Gaussian distribution on $\mathbb{R}$. Then

(a) $\mathbb{E} W_{1}\left(\mu_{n}, \gamma\right) \leq \frac{C}{n^{1 / 6}}$

(b) $\mathbb{P}\left[W_{1}\left(\mu_{n}, \gamma\right) \geq \frac{C}{n^{1 / 6}}+t\right] \leq e^{-9 n t^{2} / 2}$, and

(c) with probability 1 , for all sufficiently large $n$,

$$
W_{1}\left(\mu_{n}, \gamma\right) \leq \frac{C^{\prime}}{n^{1 / 6}}
$$

Because the coefficients $Z_{a, b, j}$ in (15) are taken to be i.i.d. Gaussian random variables, the Gaussian concentration of measure phenomenon (Proposition 2) can be combined with Lemma 10 to carry out a version of the approach used in the cases of random sums and random compressions (Sections 5 and 6). The following lemma provides the necessary link between Lemma 1 and Proposition 2 for this random matrix model.

Lemma 18. Let $\mathbf{x}=\left\{x_{a, b, j}\right\} \in \mathbb{R}^{9 n}$ (with, say, lexicographic ordering), and assume that $n \geq 3$. Define $H_{n}(\mathbf{x})$ by

$$
H_{n}(\mathbf{x}):=\frac{1}{3 \sqrt{n}} \sum_{a, b=1}^{3} \sum_{j=1}^{n} x_{a, b, j} \sigma_{j}^{(a)} \sigma_{j+1}^{(b)} .
$$

Then the map $\mathbf{x} \mapsto H_{n}$ is $\frac{2^{n / 2}}{3 \sqrt{n}}$-Lipschitz. 
Lemma 18 and Lemma 1 (b) together show that

$$
\mathbf{x} \mapsto W_{1}\left(\mu_{n}, \gamma\right)
$$

is a $\frac{1}{3 \sqrt{n}}$-Lipschitz function of $\mathbf{x}$. Part (b) of Theorem 17 then follows from part (a) and Proposition 2, and part (c) follows by the Borel-Cantelli lemma.

The proof of part (a) has two main components. First, $W_{1}\left(\mu_{n}, \mathbb{E} \mu_{n}\right)$ is estimated via the approach used in Sections 5 and 6. Lemma 18, Lemma [1](a), and Proposition 2 show that the stochastic process

$$
X_{f}:=\int f d \mu_{n}-\mathbb{E} \int f d \mu_{n}
$$

satisfies a subgaussian increment condition as in Lemma 15, which can then be used to show that.

$$
\mathbb{E} W_{1}\left(\mu_{n}, \mathbb{E} \mu_{n}\right) \leq \frac{C}{n^{1 / 6}} .
$$

Second, the convergence in expectation proved in [27] was done via a pointwise estimate of the difference between the characteristic functions of $\mathbb{E} \mu_{n}$ and $\gamma$; this estimate can be parlayed into an estimate on $W_{1}\left(\mathbb{E} \mu_{n}, \gamma\right)$ via Fourier analysis. This is carried out in detail in [3] for the bounded-Lipschitz distance; a similar argument shows that

$$
W_{1}\left(\mathbb{E} \mu_{n}, \gamma\right) \leq \frac{C}{n^{1 / 6}},
$$

completing the proof of Theorem 17 .

\section{The complex Ginibre ensemble}

Let $G_{n}$ be an $n \times n$ random matrix with i.i.d. standard complex Gaussian entries; $G_{n}$ is said to belong to the complex Ginibre ensemble. It was first established by Mehta that if $\mu_{n}$ is the empirical spectral measure of $\frac{1}{\sqrt{n}} G_{n}$, then as $n \rightarrow \infty, \mu_{n}$ converges to the circular law; i.e., to the uniform measure $\mu$ on the unit disc $D:=\{z \in \mathbb{C}|| z \mid \leq 1\}$.

This is the one ensemble we treat in which the general concentration of measure approach does not apply. The issue is that while there is a concentration phenomenon for the i.i.d. Gaussian entries of $G_{n}$, the spectral measure of a nonnormal matrix ( $G_{n}$ is nonnormal with probability 1) is not a Lipschitz function of the matrix. Nevertheless, the eigenvalue process of $G_{n}$ is a determinantal point process, and so some of the techniques used above are still available. We sketch the basic idea below; full details can be found in [38]

The eigenvalues of $G_{n}$ form a determinantal point process on $\mathbb{C}$ with the kernel

$$
K(z, w)=\frac{1}{\pi} e^{-\left(|z|^{2}+|w|^{2}\right) / 2} \sum_{k=0}^{n-1} \frac{(z \bar{w})^{k}}{k !} .
$$

This means that in principle, the determinantal approach to eigenvalue rigidity used in the case of the GUE (Section 1) and of the compact classical groups (Section 3) can be used for this model. A challenge, however, is the lack of an obvious order on the eigenvalues of an arbitrary matrix over $\mathbb{C}$; without one, there is no hope to assign predicted locations around which the individual eigenvalues concentrate. We therefore impose an order on $\mathbb{C}$ which is well-adapted for our purposes; we refer to this as the spiral order. Specifically, the linear order $\prec$ on $\mathbb{C}$ is defined by making 0 initial, and for nonzero $w, z \in \mathbb{C}$, we declare $w \prec z$ if any of the following holds:

$$
\text { - }\lfloor\sqrt{n}|w|\rfloor<\lfloor\sqrt{n}|z|\rfloor \text {. }
$$


- $\lfloor\sqrt{n}|w|\rfloor=\lfloor\sqrt{n}|z|\rfloor$ and $\arg w<\arg z$.

- $\lfloor\sqrt{n}|w|\rfloor=\lfloor\sqrt{n}|z|\rfloor, \arg w=\arg z$, and $|w| \geq|z|$.

Here we are using the convention that $\arg z \in(0,2 \pi]$.

We order the eigenvalues according to $\prec$ : first the eigenvalues in the disc of radius $\frac{1}{\sqrt{n}}$ are listed in order of increasing argument, then the ones in the annulus with inner radius $\frac{1}{\sqrt{n}}$ and outer radius $\frac{2}{\sqrt{n}}$ in order of increasing argument, and so on. We then define predicted locations $\tilde{\lambda}_{j}$ for (most of) the eigenvalues based on the spiral order: $\tilde{\lambda}_{1}=0,\left\{\tilde{\lambda}_{2}, \tilde{\lambda}_{3}, \tilde{\lambda}_{4}\right\}$ are $\frac{1}{\sqrt{n}}$ times the $3^{\text {rd }}$ roots of unity (in increasing order with respect to $\prec$ ), the next five are $\frac{2}{\sqrt{n}}$ times the $5^{\text {th }}$ roots of unity, and so on. Letting $\nu_{n}$ denote the normalized counting measure supported on the $\left\{\tilde{\lambda}_{j}\right\}$, it is easy to show that

$$
W_{2}\left(\nu_{n}, \mu\right) \leq \frac{C}{\sqrt{n}}
$$

(In fact, there is a slight modification for about $\sqrt{n \log (n)}$ of the largest eigenvalues, the details of which we will not discuss here.)

The same type of argument as in the earlier determinantal cases gives a Bernstein-type inequality for the eigenvalue counting function on an initial segment with respect to the spiral order, which in turn leads to eigenvalue rigidity for most of the eigenvalues. The largest eigenvalues can be treated with a more elementary argument, leading via the usual coupling argument to the bound

$$
\mathbb{E} W_{2}\left(\mu_{n}, \nu_{n}\right) \leq C\left(\frac{\log (n)}{n}\right)^{1 / 4}
$$

(One can deduce a slightly tighter bound for $\mathbb{E} W_{p}\left(\mu_{n}, \nu_{n}\right)$ for $1 \leq p<2$, and a weaker one for $p>2$.)

In this setting we cannot argue that the concentration of $W_{1}\left(\mu_{n}, \mu\right)$ is immediate from general concentration properties of the ensemble, but the eigenvalue rigidity itself can be used as a substitute. Indeed,

$$
W_{2}\left(\mu_{n}, \nu_{n}\right)^{2} \leq \frac{1}{n} \sum_{j=1}^{n}\left|\lambda_{j}-\tilde{\lambda}_{j}\right|^{2}
$$

and so

$$
\mathbb{P}\left[W_{2}\left(\mu_{n}, \nu_{n}\right)^{2}>t\right] \leq \mathbb{P}\left[\sum_{j=1}^{n}\left|\lambda_{j}-\tilde{\lambda}_{j}\right|^{2}>n t\right] \leq \sum_{j=1}^{n} \mathbb{P}\left[\left|\lambda_{j}-\tilde{\lambda}_{j}\right|^{2}>t\right] .
$$

For most of the eigenvalues the eigenvalue rigidity about $\tilde{\lambda}_{j}$ is strong enough to bound this quite sharply; as before, for about $\sqrt{n \log (n)}$ of the largest eigenvalues a more trivial bound is used. Since this approach does not produce a particularly clean tail inequality for $W_{2}\left(\mu_{n}, \nu_{n}\right)$, we will instead simply state the almost-sure convergence rate which follows by the Borel-Cantelli lemma.

Theorem 19. Let $\mu_{n}$ denote the empirical spectral measure of $\frac{1}{\sqrt{n}} G_{n}$, and let $\mu$ denote the uniform measure on the unit disc in $\mathbb{C}$. Then with probability 1 , for sufficiently large $n$,

$$
W_{2}\left(\mu_{n}, \mu\right) \leq C \frac{\sqrt{\log n}}{n^{1 / 4}} .
$$




\section{ACKNowledgements}

This research was partially supported by grants from the U.S. National Science Foundation (DMS-1308725 to E.M.) and the Simons Foundation (\#315593 to M.M.). This paper is an expansion of the first-named author's talk at the excellent workshop "Information Theory and Concentration Phenomena" at the Institute for Mathematics and its Applications, as part of the IMA Thematic Year on Discrete Structures: Analysis and Applications. The authors thank the IMA for its hospitality.

\section{REFERENCES}

[1] G. W. Anderson, A. Guionnet, and O. Zeitouni. An Introduction to Random Matrices, volume 118 of Cambridge Studies in Advanced Mathematics. Cambridge University Press, Cambridge, 2010.

[2] R. Bhatia. Matrix Analysis, volume 169 of Graduate Texts in Mathematics. Springer-Verlag, New York, 1997.

[3] D. Buzinski and E. S. Meckes. Almost sure convergence in quantum spin glasses. J. Math. Phys., 56(12), 2015.

[4] S. Chatterjee. Concentration of Haar measures, with an application to random matrices. J. Funct. Anal., 245(2):379-389, 2007.

[5] S. Chatterjee and M. Ledoux. An observation about submatrices. Electron. Commun. Probab., 14:495$500,2009$.

[6] S. Dallaporta. Eigenvalue variance bounds for Wigner and covariance random matrices. Random Matrices Theory Appl., 1(3):1250007, 28, 2012.

[7] S. Dallaporta. Eigenvalue variance bounds for covariance matrices. Markov Process. Related Fields, 21(1):145-175, 2015.

[8] K. R. Davidson and S. J. Szarek. Local operator theory, random matrices and Banach spaces. In Handbook of the Geometry of Banach Spaces, Vol. I, pages 317-366. North-Holland, Amsterdam, 2001.

[9] P. Diaconis and M. Shahshahani. On the eigenvalues of random matrices. J. Appl. Probab., 31A:49-62, 1994. Studies in applied probability.

[10] R. Dudley. V. N. Sudakov's work on expected suprema of Gaussian processes. To appear in the proceedings of High Dimensional Probability VII.

[11] F. J. Dyson. Correlations between eigenvalues of a random matrix. Comm. Math. Phys., 19:235-250, 1970.

[12] L. Erdős and D. Schröder. Phase transition in the density of states of quantum spin glasses. Math. Phys. Anal. Geom., 17(3-4):441-464, 2014.

[13] L. Erdős and H.-T. Yau. Universality of local spectral statistics of random matrices. Bull. Amer. Math. Soc. (N.S.), 49(3):377-414, 2012.

[14] L. Erdős, H.-T. Yau, and J. Yin. Rigidity of eigenvalues of generalized Wigner matrices. Adv. Math., $229(3): 1435-1515,2012$.

[15] L. C. Evans and R. F. Gariepy. Measure theory and fine properties of functions. Studies in Advanced Mathematics. CRC Press, Boca Raton, FL, 1992.

[16] P. J. Forrester and E. M. Rains. Interrelationships between orthogonal, unitary and symplectic matrix ensembles. In Random matrix models and their applications, volume 40 of Math. Sci. Res. Inst. Publ., pages 171-207. Cambridge Univ. Press, Cambridge, 2001.

[17] F. Götze and A. Tikhomirov. Optimal bounds for convergence of expected spectral distributions to the semi-circular law. To appear in Probab. Theory Related Fields.

[18] F. Götze and A. Tikhomirov. The rate of convergence for spectra of GUE and LUE matrix ensembles. Cent. Eur. J. Math., 3(4):666-704 (electronic), 2005.

[19] N. Gozlan. A characterization of dimension free concentration in terms of transportation inequalities. Ann. Probab., 37(6):2480-2498, 2009.

[20] N. Gozlan, C. Roberto, and P.-M. Samson. From dimension free concentration to the Poincaré inequality. Calc. Var. Partial Differential Equations, 52(3-4):899-925, 2015.

[21] M. Gromov and V. D. Milman. A topological application of the isoperimetric inequality. Amer. J. Math., 105(4):843-854, 1983. 
[22] A. Guionnet and O. Zeitouni. Concentration of the spectral measure for large matrices. Electron. Comm. Probab., 5:119-136 (electronic), 2000.

[23] J. Gustavsson. Gaussian fluctuations of eigenvalues in the GUE. Ann. Inst. H. Poincaré Probab. Statist., 41(2):151-178, 2005.

[24] J. B. Hough, M. Krishnapur, Y. Peres, and B. Virág. Determinantal processes and independence. Probab. Surv., 3:206-229, 2006.

[25] V. Kargin. A concentration inequality and a local law for the sum of two random matrices. Probab. Theory Related Fields, 154(3-4):677-702, 2012.

[26] N. M. Katz and P. Sarnak. Random Matrices, Frobenius Eigenvalues, and Monodromy, volume 45 of American Mathematical Society Colloquium Publications. American Mathematical Society, Providence, RI, 1999.

[27] J. P. Keating, N. Linden, and H. J. Wells. Spectra and eigenstates of spin chain Hamiltonians. Comm. Math. Phys., 338(1):81-102, 2015.

[28] M. Ledoux. The Concentration of Measure Phenomenon, volume 89 of Mathematical Surveys and Monographs. American Mathematical Society, Providence, RI, 2001.

[29] M. Ledoux. Deviation inequalities on largest eigenvalues. In Geometric Aspects of Functional Analysis, volume 1910 of Lecture Notes in Math., pages 167-219. Springer, Berlin, 2007.

[30] M. Ledoux. $\gamma_{2}$ and $\Gamma_{2}$. Unpublished note, available at http://perso.math.univ-toulouse.fr/ledoux/files/2015/06/gGamma2.pdf, 2015.

[31] M. Ledoux and B. Rider. Small deviations for beta ensembles. Electron. J. Probab., 15:no. 41, 1319-1343, 2010.

[32] V. A. Marčenko and L. A. Pastur. Distribution of eigenvalues in certain sets of random matrices. Mat. Sb. (N.S.), 72 (114):507-536, 1967.

[33] E. Meckes. Approximation of projections of random vectors. J. Theoret. Probab., 25(2):333-352, 2012.

[34] E. Meckes. Projections of probability distributions: a measure-theoretic Dvoretzky theorem. In Geometric Aspects of Functional Analysis, volume 2050 of Lecture Notes in Math., pages 317-326. Springer, Heidelberg, 2012.

[35] E. S. Meckes and M. W. Meckes. Another observation about operator compressions. Proc. Amer. Math. Soc., 139(4):1433-1439, 2011.

[36] E. S. Meckes and M. W. Meckes. Concentration and convergence rates for spectral measures of random matrices. Probab. Theory Related Fields, 156(1-2):145-164, 2013.

[37] E. S. Meckes and M. W. Meckes. Spectral measures of powers of random matrices. Electron. Commun. Probab., 18:no. 78, 13, 2013.

[38] E. S. Meckes and M. W. Meckes. A rate of convergence for the circular law for the complex Ginibre ensemble. Ann. Fac. Sci. Toulouse Math. (6), 24(1):93-117, 2015.

[39] M. L. Mehta. Random Matrices, volume 142 of Pure and Applied Mathematics (Amsterdam). Elsevier/Academic Press, Amsterdam, third edition, 2004.

[40] S. Ng and M. Walters. A method to derive concentration of measure bounds on Markov chains. Electron. Comm. Probab., 20(95), 2015.

[41] S. O'Rourke. Gaussian fluctuations of eigenvalues in Wigner random matrices. J. Stat. Phys., 138(6):1045-1066, 2010.

[42] E. M. Rains. Images of eigenvalue distributions under power maps. Probab. Theory Related Fields, 125(4):522-538, 2003.

[43] R. Speicher. Free convolution and the random sum of matrices. Publ. Res. Inst. Math. Sci., 29(5):731744, 1993.

[44] M. Talagrand. Upper and Lower Bounds for Stochastic Processes: Modern Methods and Classical Problems, volume 60 of Ergebnisse der Mathematik und ihrer Grenzgebiete. 3. Folge. Springer, Heidelberg, 2014.

[45] T. Tao and V. Vu. Random matrices: universality of local eigenvalue statistics up to the edge. Comm. Math. Phys., 298(2):549-572, 2010.

[46] T. Tao and V. Vu. Random matrices: universality of local eigenvalue statistics. Acta Math., 206(1):127204, 2011.

[47] T. Tao and V. Vu. Random matrices: sharp concentration of eigenvalues. Random Matrices Theory Appl., 2(3):1350007, 31, 2013. 
[48] J. A. Tropp. An introduction to matrix concentration inequalities. Foundations and Trends in Machine Learning, 8(1-2), 2015.

[49] R. Vershynin. Introduction to the non-asymptotic analysis of random matrices. In Compressed Sensing, pages 210-268. Cambridge Univ. Press, Cambridge, 2012.

[50] C. Villani. Topics in Optimal Transportation, volume 58 of Graduate Studies in Mathematics. American Mathematical Society, Providence, RI, 2003.

[51] D. Voiculescu. Limit laws for random matrices and free products. Invent. Math., 104(1):201-220, 1991.

[52] E. Wigner. Characteristic vectors of bordered matrices with infinite dimensions. Ann. of Math. (2), 62:548-564, 1955.

[53] E. Wigner. On the distribution of the roots of certain symmetric matrices. Ann. of Math. (2), 67:325$327,1958$.

Department of Mathematics, Applied Mathematics, and Statistics, Case Western Reserve University, 10900 Euclid Ave., Cleveland, Ohio 44106, U.S.A.

E-mail address: elizabeth.meckes@case.edu

Department of Mathematics, Applied Mathematics, and Statistics, Case Western Reserve

University, 10900 Euclid Ave., Cleveland, Ohio 44106, U.S.A.

E-mail address: mark.meckes@case.edu 